\title{
Distributed User Selection in Network MIMO Systems with Limited Feedback
}

\author{
Khalil Elkhalil ${ }^{1}$, Mohammed E. Eltayeb ${ }^{2}$, Hayssam Dahrouj ${ }^{3}$ and Tareq Y. Al-Naffouri ${ }^{1}$ \\ ${ }^{1}$ Electrical Engineering Department, King Abdullah University of Science and Technology, Thuwal, Saudi Arabia. \\ ${ }^{2}$ Department of Electrical and Computer Engineering, The University of Texas at Austin, Texas, USA. \\ ${ }^{3}$ Department of Electrical and Computer Engineering, Effat University, Jeddah, KSA. \\ E-mails: \{khalil.elkhalil, tareq.alnaffouri\}@kaust.edu.sa, meltayeb@utexas.edu, hayssam.dahrouj@gmail.com
}

\begin{abstract}
We propose a distributed user selection strategy in a network MIMO setting with $M$ base stations serving $K$ users. Each base station is equipped with $L$ antennas, where $L M \ll K$. The conventional selection strategy is based on a well known technique called semi-orthogonal user selection when the zero-forcing beamforming (ZFBF) is adopted. Such technique, however, requires perfect channel state information at the transmitter (CSIT), which might not be available or need large feedback overhead. This paper proposes an alternative distributed user selection technique where each user sets a timer that is inversely proportional to his channel quality indicator (CQI), as a means to reduce the feedback overhead. The proposed strategy allows only the user with the highest CQI to respond with a feedback. Such technique, however, remains collision free only if the transmission time is shorter than the difference between the strongest user timer and the second strongest user timer. To overcome the situation of longer transmission times, the paper proposes another feedback strategy that is based on the theory of compressive sensing, where collision is allowed and all users encode their feedback information and send it back to the basestations simultaneously. The paper shows that the problem can be formulated as a block sparse recovery problem which is agnostic on the transmission time, which makes it a good alternative to the timer approach when collision is dominant.

Index Terms-Network MIMO, Distributed user selection, limited feedback, Collision, Compressive Sensing.
\end{abstract}

\section{INTRODUCTION}

In network multiple input multiple output (NW-MIMO) systems where base- stations (BSs) jointly coordinate to transmit their signals to a selected pool of users, the network throughput can be significantly improved by minimizing inter-cell interference (ICI). Significant improvement can be also achieved when considering efficient multiuser scheduling schemes that aim to bound the feedback overhead while maximizing the downlink capacity [1].

With a NW-MIMO system with $M$ BSs equipped with $L$ antennas each, and $K$ users, full multiplexing gain of $L M$ can be achieved using space-division multiple access schemes such as dirty-paper coding that treats the system as a conventional MIMO system. The gain achieved by MIMO systems is promising; however, it comes with high complexity. The complexity becomes even higher with NW-MIMO systems since all BSs have to coordinate in order to find the optimal set of users that maximize the sum-rate; thus, the need of distributed schemes to schedule users and to design their beamforming vectors. Low complexity schemes such as zero-forcing beamforming ( $\mathrm{ZFBF})$ that can tolerate some performance loss can also be implemented and asymptotically achieves the same $L M \log \log K$ growth in sum-rate when the number of users is large [2].

However, most of the previous schemes assume perfect channel state information at the transmitter (CSIT), which is not realistic in practical systems. In [3], the authors prove that with a finite feedback rate, the system can have comparable performance to the perfect CSIT case in terms of sum rate. It is also proven that the number of feedback bits required for each user to quantize its channel direction indicator (CDI) decreases with the number of users. While this may reduce the feedback per user, the overall feedback rate increases linearly with $K$ since each user has to encode its CDI individually.

In this paper, we propose a distributed user selection strategy that achieves comparable performance in terms of sum-rate while reducing the overall feedback overhead. The paper solves the problem using two approaches:

- Timer-based approach: users are selected based on a metric inversely proportional to their channels norms or channel quality indicator (CQI) and only the users with the highest CQI is allowed to feedback. This approach is successful as long as there is no collision in the system. In the case of collision, the BSs are not able to decode the fed back information and an outage is declared.

- Compressive Sensing (CS)-based approach: Unlike the timer approach, in the CS approach users are allowed to feedback simultaneously and collsion is resolved using compressive sensing. More precisely, only a pool of users having their CQI higher than a certain threshold are allowed to feedback using signature sequences at the same time. Since the fed back vector is sparse, the BSs can apply sparse recovery algorithm to recover the identity and the CQI of the strongest user.

Simulations results show that on one hand, the performance of the timer-based scheme is sensible to the transmission duration and that the sum-rate deteriorates when the transmission time is large. On the other hand, the CS-based scheme is agnostic on the transmission time and can be an alternative to the timerbased scheme when this quantity is large. Moreover, we prove that the feedback load for both the timer-based approach and the CS-based approach is much reduced as compared to the 


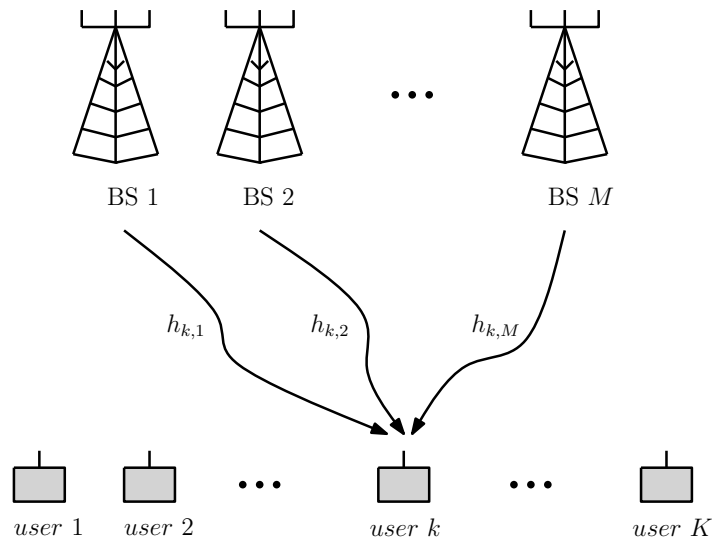

Figure 1. Network MIMO system with $M$ BSs and $K$ users.

perfect CSIT case, and grows logarithmically with $K$, i.e. scales as $\mathcal{O}(\log K)$.

The remainder of the paper is organized as follows. In section II, we introduce the network model. In section III, we present the timer-based approach and the CS-based approach. In section IV, we evaluate the performance of the proposed feedback algorithms. In section V, we provide some numerical results and we conclude our work in section VI.

\section{Network Model}

Consider a network consisting of $M$ base-stations (BSs) serving $K$ single antenna users. Each BS is equipped with $L$ antennas. The paper assumes that the number of users is higher than the total number of antennas over the network, i.e. $K \geq L M$. The received signal by the $k$ th user is given by

$$
y_{k}=\mathbf{h}_{k} \mathbf{x}+z_{k}, \quad k=1,2, \ldots, K,
$$

where $\mathbf{h}_{k}=\left[h_{k, 1}, h_{k, 2}, \ldots, h_{k, L M}\right] \in \mathbb{C}^{1 \times L M}$ is the complex channel vector between all the BSs and the $k$ th user, and $h_{k, l}$ is the channel between the $k$ th user and the $l$ th antenna, where $l \in\{1, \cdots, L M\}, \mathbf{x}$ is the transmitted signal and $z_{k} \sim C \mathcal{N}(0,1)$ the complex additive noise. The entries of $\mathbf{H}=\left[\mathbf{h}_{1}^{T}, \mathbf{h}_{2}^{T}, \ldots, \mathbf{h}_{K}^{T}\right] \in \mathbb{C}^{L M \times K}$ are assumed to be i.i.d Gaussian with zero mean and unit variance. We denote by $\mathcal{K}=\{\pi(1), \pi(2), \ldots, \pi(|\mathcal{K}|)\}$ the set of selected users and by $s_{k}, k \in \mathcal{K}$ the information symbols. Then, the transmitted signal vector $\mathrm{x}$ is produced using linear beamforming of the information symbols as $\mathbf{x}=\sum_{k \in \mathcal{K}} \sqrt{P_{k}} \mathbf{w}_{k} s_{k}$ with an average power constraint $E\left[\|\mathbf{x}\|^{2}\right]=P$. Therefore, for a selected user $k \in \mathcal{K}$, the received signal can be expressed as

$$
y_{k}=\sqrt{P_{k}}\left(\mathbf{h}_{k} \mathbf{w}_{k}\right) s_{k}+\sum_{j \in \mathcal{K}, j \neq k} \sqrt{P_{j}}\left(\mathbf{h}_{k} \mathbf{w}_{j}\right) s_{j}+z_{k},
$$

where $\mathcal{K}$ and the set of beamformers $\left\{\mathbf{w}_{k} \in \mathbb{C}^{L M \times 1}, k \in \mathcal{K}\right\}$ are chosen to maximize the sum-rate and $P_{k}$ is the transmit power for the $k$ th selected user. Among the linear beamforming strategies, we consider zero-forcing beamforming (ZFBF) where the beamforming vectors are constructed to satisfy $\mathbf{h}_{k} \mathbf{w}_{l}=0, \forall l, k \in \mathcal{K}$ with $l \neq k$. Therefore, the beamforming vectors are given by the columns of the pseudo-inverse

$$
\mathbf{W}(\mathcal{K})=\mathbf{H}(\mathcal{K})^{H}\left(\mathbf{H}(\mathcal{K}) \mathbf{H}(\mathcal{K})^{H}\right)^{-1}
$$

where $\mathbf{w}_{k}$ the $k$ th column of $\mathbf{W}(\mathcal{K})$ is the beamforming vector for the $k$ th selected user. The scheduler selects a set of users and construct their beamforming vectors using ZFBF to support up to $M$ users. However, finding the optimal set of users that maximize the sum-rate is computationally unfeasible for moderate to large $K$. Instead, heuristic user selection algorithms can be applied to reduce the search complexity with a tolerable loss in performance. In the following section, we present a limited feedback scheduling algorithm based on semi-orthogonal user selection (SUS) technique [2], [4].

\section{SEMI-ORTHOGONAL USER SELECTION WITH LIMITED FEEDBACK}

Without loss of generality, in the remaining of this paper, we assume that the base-stations are equipped with single antenna each, i.e. $L=1$ as shown in Fig. 1, however all the results can easily be extended to the multiple antennas case. The intuition behind this algorithm is that if the selected users are mutually semi-orthogonal, the ZFBF scheme achieves optimality in terms of sum-rate [2]. Based on this, the scheduler can select users that have good channel qualities and are mutually semi-orthogonal. If we assume that the scheduler has perfect channel state information of the users (perfect CSIT), the SUS procedure is summarized as follows $[2,3]$

- The scheduler starts by selecting the user with the highest channel norm :

$$
\pi(1)=\underset{k \in\{1,2, \ldots, K\}}{\operatorname{argmax}}\left\|\mathbf{h}_{k}\right\|^{2}
$$

- Select the $(i+1)$ th user as

$$
\pi(i+1)=\underset{k \in \mathcal{A}_{i}}{\operatorname{argmax}}\left\|\mathbf{h}_{k}\right\|^{2},
$$

where $\mathcal{A}_{i}=\left\{1 \leq k \leq K: \frac{\left|\mathbf{h}_{k} \mathbf{h}_{\pi(j)}^{H}\right|}{\left\|\mathbf{h}_{k}\right\|\left\|\mathbf{h}_{\pi(j)}\right\|} \leq \epsilon, 1 \leq j \leq i\right\}$ and $i \leq M-1$

\section{A. Proposed distributed user selection algorithm}

For the scheduler to execute the SUS algorithm, it is assumed that perfect CSIT is available. However, this assumption is not valid for practical scenarios. Instead, each user $k$ can feedback its channel direction information (CDI) $\mathbf{h}_{k} /\left\|\mathbf{h}_{k}\right\|$ using $B$ bits quantization and its unquantized channel quality indicator (CQI) $\left\|\mathbf{h}_{k}\right\|^{2}$. It has been shown in [2], that having more users decreases the amount of feedback bits needed per user. However, the overall feedback load still scales linearly with $K$, since each user has to feedback its quantized CDI independently and no contention is allowed. Motivated by this problem, we propose a limited feedback strategy that allows distributed user selection based on the SUS algorithm described previously. The idea behind our algorithm is simple and is based on the observation that it is not necessary to 
request feedback from all users and only a pool of users satisfying good CQI requirements and mutual semi-orthogonality are allowed to feedback. The proposed feedback algorithm is then described as follows

1) Each BS broadcasts a pilot in an independent channel. This allows each user to estimate its channel of length $M$.

2) Each user $k$ waits for one mini slot and calculate its CQI, $\left\|\mathbf{h}_{k}\right\|^{2}$ and sets a timer $\tau(k)=\frac{T_{m s}}{\left\|\mathbf{h}_{k}\right\|^{2}}$, where $T_{m s}$ is the mini-slot duration ${ }^{1}$. Using this timer, each user waits until its timer expires before feedback. Thus, the user having the highest CQI feedbacks the first. The remaining users overhear the transmission of that user and they back off. The feedback information consists of the identity (ID) of the selected user and a pilot normalized by its channel norm (each of these information consumes one mini slot), thus the lth BS can estimate the quantity $\frac{h_{\pi(i), l}}{\left\|\mathbf{h}_{\pi(i)}\right\|}, \pi(i)$ is the index of the selected user.

3) Assuming that we have already scheduled $i$ users. Using the estimated quantity from the previous step, each BS $l$, transmits $h_{\pi(i), l}^{*} /\left\|\mathbf{h}_{\pi(i)}\right\|$ along with the other BSs. Thus, after normalizing with its channel norm, each user $j$ receives $c_{j}=\sum_{l=1}^{L M} \frac{h_{j, l} h_{\pi(i), l}^{*}}{\left\|\mathbf{h}_{j}\right\|\left\|\mathbf{h}_{\pi(i)}\right\|}=\frac{\mathbf{h}_{j} \mathbf{h}_{\pi(i)}^{H}}{\left\|\mathbf{h}_{j}\right\|\left\|\mathbf{h}_{\pi(i)}\right\|}$. Now, each user is able to compute its correlation with the previously selected users. Only users satisfying the semiorthogonality requirement $\left(\left|c_{j}\right| \leq \epsilon\right)$ are allowed to set a timer, then repeat III.B. step 2). In the case where no user feedback, the BSs can not wait indefinitely, thus we select one base-station to control this event ${ }^{2}$. To do that, if a user is found to have a correlation less than $\epsilon$, it sends a "1" bit notifier in the first mini slot. Thus, if the pre-selected BS receives a sum equal to $K-i$, it terminates the scheduling process, otherwise repeat III.B. step 3) until $M$ users are scheduled.

\section{B. Proposed distributed user selection algorithm: Effect of collision}

In the previous subsection, in III.B. step 2) we assume that the remaining users back off when they overhear the fed back information from the strongest user. However, this implicitly assumes that the time to feedback is negligible so that no collision occurs between users. This assumption is not realistic and the feedback time has to be taken into account. Let $\lambda T_{m s}$ be the time needed to communicate the fed back information where $\lambda$ is assumed to be a constant. Then no collision occurs if and only if the timers of the best and the second best users expires within a duration not less then $\lambda T_{m s}$. For simplicity assume that the index of the best and the second best user are (1) and (2) respectively, then the probability of collision is

\footnotetext{
${ }^{1}$ The idea of setting a timer based on the CQI has been investigated in the context of cooperative relay networks [5]

${ }^{2}$ This BS is selected to check if the users are semi-orthogonal to the scheduled users and does not need to coordinate with other BSs.
}

given by

$$
\begin{aligned}
\mathcal{P}_{\text {collision }} & =\mathbb{P}\left\{\frac{T_{m s}}{\left\|\mathbf{h}_{(2)}\right\|^{2}}-\frac{T_{m s}}{\left\|\mathbf{h}_{(1)}\right\|^{2}}<\lambda T_{m s}\right\} \\
& =\mathbb{P}\left\{\frac{1}{\left\|\mathbf{h}_{(2)}\right\|^{2}}-\frac{1}{\left\|\mathbf{h}_{(1)}\right\|^{2}}<\lambda\right\}
\end{aligned}
$$

\section{Distributed user selection: Compressive Sensing Approach}

In the previous algorithm, users have to wait until their timers expire. However, this may result in a collision as stated before, and an outage may occur from the first iteration. Instead, we propose a Compressive Sensing based feedback algorithm, where we embrace collision and allow some users to feedback at the same time [6],[7]. Indeed, we set a threshold to sparsify users, i.e. only users having a channel CQI higher than a predetermined threshold are allowed to feedback at each iteration. Starting from the second iteration, active users should also satisfy the semi-orthogonality requirement. At each iteration, a feedback threshold is calculated to yield small outage probability. One way to set the threshold $\gamma_{i}$ is the following

$$
\left[F_{\|\mathbf{h}\|^{2}}\left(\gamma_{i}\right)\right]^{K-i+1}=\mathcal{P}_{0}
$$

where $\mathcal{P}_{0}$ is a target outage probability and $F_{\|\mathbf{h}\|^{2}}($.$) is the$ $\mathrm{CDF}$ of the vector channel norm square of an arbitrary user. In the following, we show how we can exploit useful results from block sparse recovery literature in order to schedule users in the network MIMO setting using ZFBF and approach the performance of the SUS algorithm with much reduced feedback overhead. The proposed CS-based selection algorithm is described as follows

1) Each user in the system estimates its CDI and its CQI using training from each antenna independently.

2) For the $i$ th user selection, we assume that each user is assigned two code words of length $J$ to be used in the feedback period. Prior to feedback, each user compares its CQI with $\gamma_{i}$ and combines its CQI and a known symbol $\mathbf{s}$ with its allocated feedback code and transmits the combination to all the BSs,,i.e. at the $m$ th feedback slot, user $k$ transmits $a_{m, 2 k-1} \mathbf{s}+a_{m, 2 k}\left\|\mathbf{h}_{k}\right\|^{2}$, if its CQI is higher than $\gamma_{i}{ }^{3}$ :

$$
\begin{aligned}
& \text { if }\left\|\mathbf{h}_{k}\right\|^{2} \geq \gamma_{i} \text { then } \\
& \quad \quad \operatorname{transmit}\left(a_{m, 2 k-1} \mathbf{s}+a_{m, 2 k}\left\|\mathbf{h}_{k}\right\|^{2}\right), \\
& \text { for } m=1,2, \ldots, J \\
& \text { else } \\
& \quad \text { be silent }
\end{aligned}
$$

Therefore, each BS $l$ receives $\mathbf{y}_{l}=\mathbf{A}\left(\mathbf{g}_{l} \circ \mathbf{X}\right)+\mathbf{z}_{l}$, where $\circ$ denotes the Hadamard product, $\mathbf{A}=\left(a_{m, p}\right)_{1 \leq m \leq J, 1 \leq p \leq 2 K}$ is a real Gaussian matrix with zero mean and variance $1 / J$, $\mathbf{g}_{l} \quad=\quad\left[h_{1, l}, h_{1, l}, h_{2, l}, h_{2, l}, \ldots, h_{K, l}, h_{K, l}\right]^{T}$,

\footnotetext{
${ }^{3}$ Note that starting from the second iteration, the users are naturally sparse since few of them are semi-orthogonal to the previously scheduled users. Therefore, we can ignore the comparison with a threshold before feedback.
} 
$\mathbf{X}=\left[\mathbf{s},\left\|\mathbf{h}_{1}\right\|^{2}, \mathbf{s},\left\|\mathbf{h}_{2}\right\|^{2}, \ldots, \mathbf{s},\left\|\mathbf{h}_{K}\right\|^{2}\right]^{T} \quad$ and $\mathbf{z}_{l} \sim C \mathcal{N}\left(\mathbf{0}, \mathbf{I}_{J}\right)$ the additive noise.

Obviously, the fed back vector $\mathbf{x}$ is block sparse of block size 2. Using this observation, each BS $l$ can apply block CS recovery and recover the index of the strongest user $\pi(i)^{4}$ and its channel gain with it, $h_{\pi(i), l}$. If No user is detected, an outage is declared, and the scheduling is terminated.

3) Assuming that $i$ users are already scheduled, form the previous iteration, we have an estimate of $h_{l, \pi(i)}, \hat{h}_{l, \pi(i)}$ using CS. Therefore, we repeat the same treatment in III.B 2) by replacing the value of $h_{l, \pi(i)}$ by $\hat{h}_{l, \pi(i)}$ and users satisfying the semi-orthogonality requirement feedback as in III.D 2) to schedule the user for the next iteration.

4) repeat the previous steps until outage or reach $M$ scheduled users.

\section{PERformance AnAlysis}

\section{A. Achievable sum-rate}

1) Timer-based approach: Using this approach and in the case where the collision probability is negligible, the transmitter can perfectly obtain the set of active users as in the SUS algorithm [3]. However, this is not the case, when the collision probability is not negligible. The generic expression for the sum-rate is given by

$$
\begin{aligned}
\mathcal{R}_{\text {timer }} & =\sum_{k=1}^{|\mathcal{K}|} \log _{2}\left(1+P_{\pi(k)}\right)\left(1-\mathcal{P}_{\text {collision }}(k)\right) \\
& \times \prod_{i=1}^{k-1}\left(1-\mathcal{P}_{\text {collision }}(i)\right)
\end{aligned}
$$

where $\mathcal{P}_{\text {collision }}(i)$ is the collision probability at the $i$ th iteration and $P_{\pi(k)}$ is the allocated power for the $k$ th selected user [2].

2) CS-based approach: In this scheme, we deal with noisy feedback, therefore the set of the selected users may not be the true set, hence the beamforming vectors can be constructed based on a wrong information on the channel vectors. We denote by $\mathcal{K}^{\prime}$ the estimated set of users using this approach, therefore the sum-rate can be expressed as

$$
\mathcal{R}_{c s}=\sum_{k \in \mathcal{K}^{\prime}} \log _{2}\left(1+\frac{P_{k}\left|\mathbf{h}_{k} \hat{\mathbf{w}}_{k}\right|^{2}}{1+\sum_{j \in \mathcal{K}^{\prime}, j \neq k} P_{j}\left|\mathbf{h}_{k} \hat{\mathbf{w}}_{j}\right|^{2}}\right),
$$

where $\hat{\mathbf{w}}_{k}$ is the beamforming vector for the $k$ th selected user using the CS-approach.

\section{B. Feedback overhead}

1) Timer-based approach: The feedback overhead for the timer-based selection algorithm consists of two quantities : 1) waiting time: duration for the strongest user's timer to expire and 2) the transmission time: time for the selected user to

\footnotetext{
${ }^{4}$ Since the CS recovery is noisy, this might not be the true index, but for simplicity we keep the same notation.
}

transmit his identity (ID) and a pilot. Since, there are $K$ users in the system, $\log _{2} K$ bits are needed to communicate the ID of the selected user. Assume that one mini slot of duration $T_{m s}$ can transmit $n$ bits, therefore the ID of a user needs $\frac{\log _{2} K}{n}$ time-slots to be communicated. Also, note that the average number of selected users denoted by $\overline{\mathcal{K}}$ is an important metric to derive the average total feedback time.

$$
\begin{aligned}
\overline{\mathcal{K}} & =1+\sum_{k=1}^{M-1} 1-\left(1-I_{\epsilon^{2}}(k, M-k)\right)^{K-k} \\
& =M-\sum_{k=1}^{M-1}\left(1-I_{\epsilon^{2}}(k, M-k)\right)^{K \cdot-k}
\end{aligned}
$$

This directly follows from the fact that at iteration $k+1$, the probability that a user is semi-orthogonal to the previously selected users $\{\pi(k), \pi(k-1), \ldots, \pi(1)\}$ is given by $I_{\epsilon^{2}}(k, M-k)=\frac{B_{\epsilon^{2}}(k, M-k)}{B(k, M-k)}$ [4], where $B_{z}(a, b)$ is the incomplete beta function and $B(a, b)$ is the complete beta function. We denote by $F_{\text {timer }}$, the total feedback air-time needed, then

$$
\begin{aligned}
F_{\text {timer }} & =E\left\{\sum_{k=1}^{|\mathcal{K}|} T_{m s}+T_{m s}+\frac{\log _{2} K}{n} T_{m s}+2 \lambda T_{m s}+\frac{T_{m s}}{\left\|\mathbf{h}_{\pi(k)}\right\|^{2}}\right\} \\
& =\left(2+\frac{\log _{2} K}{n}+2 \lambda\right) \overline{\mathcal{K}} T_{m s}+\sum_{k=1}^{|\mathcal{K}|} E\left\{\frac{1}{\left\|\mathbf{h}_{\pi(k)}\right\|^{2}}\right\} T_{m s}
\end{aligned}
$$

As shown in the previous equation, the feedback load consists of four terms : the first term is the time needed for the preselected BS to figure out if there is an outage or not, the second term is the time needed for the selected user to communicate a pilot and his ID respectively, the third and the last terms stands for the propagation and the waiting times respectively. Clearly, the feedback load for the timer based approach grows logarithmically with $K, F_{\text {timer }}=\mathcal{O}(\log K)$.

2) CS-based approach: As stated before, each user encodes his feedback information consisting of a known symbol and his CQI and transmits his approach depends on the value of $J$. Since the fed-back vector is block sparse with block size $B=2$ and sparsity $S^{5}$, we can use the result in [8] where the authors proved that efficient CS recovery can be achieved with a number of measurements $J=\mathcal{O}\left(B S+S \log \frac{2 K}{S}\right)$. Therefore, the total feedback time for the CS-based approach to make a selection decision is

$$
\begin{aligned}
F_{c s} & =\overline{\mathcal{K}} J T_{m s} \\
& =C \overline{\mathcal{K}}\left(B S+S \log \frac{2 K}{S}\right) T_{m s} \\
& \leq C M\left(B S+S \log \frac{2 K}{S}\right) T_{m s},
\end{aligned}
$$

where the inequality directly follows from the fact that $\overline{\mathcal{K}} \leq$ $M, C$ is a positive constant and $S=K\left(1-\mathcal{P}_{0}^{1 / K}\right)$ is the

\footnotetext{
${ }^{5}$ The sparsity $S$ is the average number of non zero entries in the fed-back vector $\mathbf{X}$.
} 
average number of users that feedback at each iteration. It is clear from the previous equation that the feedback overhead for the CS-based selection algorithm grows logarithmically with the number of users $K, F_{c s}=\mathcal{O}(\log K)$.

\section{Numerical Results AND Discussion}

In this section, we provide simulation results on the performance of the two proposed selection algorithms in terms of sum-rate and feedback time. For that, we set $M=6$ BSs, $\epsilon=0.25, n=5$ bits, and $\mathcal{P}_{0}=0.01$. As shown in Figure 2, the timer-based algorithm achieves the performance of the perfect CSIT case in terms of sum-rate when $\lambda$ is close to zeros, this is the no collision case where the timerbased algorithm is equivalent to the SUS algorithm with perfect CSIT. However, the performance deteriorates when the propagation time $\lambda T_{m s}$ increases since the collision is no longer negligible and the scheduler fails to determine the identity of the strongest user. Instead, the CS-based algorithm is shown to be agnostic on the propagation delay since it allows collision between users and yields a small hit in the sum-rate performance due to noisy feedback as compared to the perfect CSIT case. In Figure 3, we compare the feedback load of the proposed selection algorithms. Both the timer and the CSbased algorithms consume much less feedback as compared to the perfect CSIT case especially at high number of users and provide a feedback load that grows logarithmically with $K$ rather than linear as in the case of the perfect CSIT. However, for a practical system where the users might be hidden or far from each other and so the feedback information from the strongest user might not reach all users, the CS solution seems to be a practical one since it is agnostic on the propagation delay and deals with noisy feedback.

\section{CONCLUSION}

In this paper, we propose a distributed user selection strategy in a multiuser network MIMO setting with $M$ BSs and $K$ users. Two approaches have been proposed. The first one is a timer-based approach where users are selected based on a metric inversely proportional to their CQI. The second one is a CS-based approach, where a pool of users selected based on a threshold are allowed to feedback at the same time after they encode their feedback information with a Gaussian code, and then each BS performs block CS recovery algorithms to recover the feedback information. The timer-based approach achieves the perfect CSIT performance in terms of sum-rate when the transmission time is small. However, when this time is not negligible, the performance of the timer-based approach deteriorates due to the increased collision in the system. The CS-based approach, however is agnostic on the transmission time and achieves a sum-rate that is close to the one achieved when perfect CSIT are available. Both approaches, consume much less feedback time as compared to perfect CSIT case and both schemes provide a feedback load that is logarithmic in $K$.

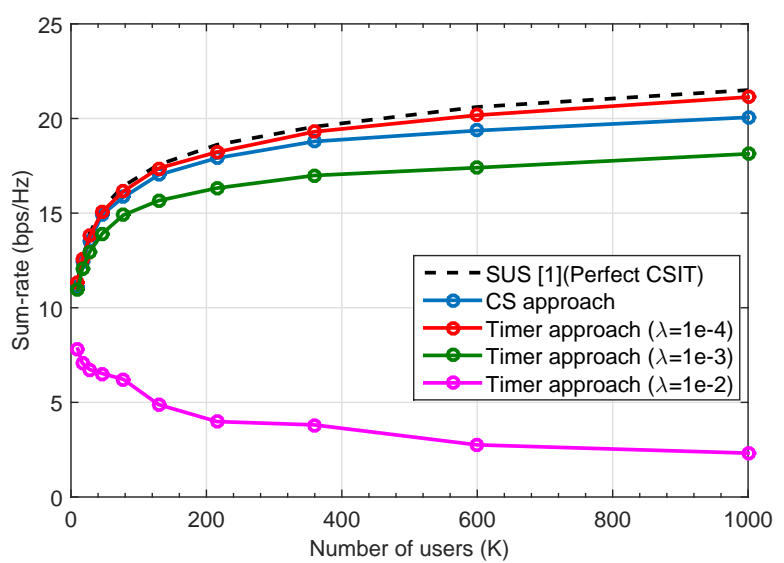

Figure 2. Sum-rate versus the number of users $K$.

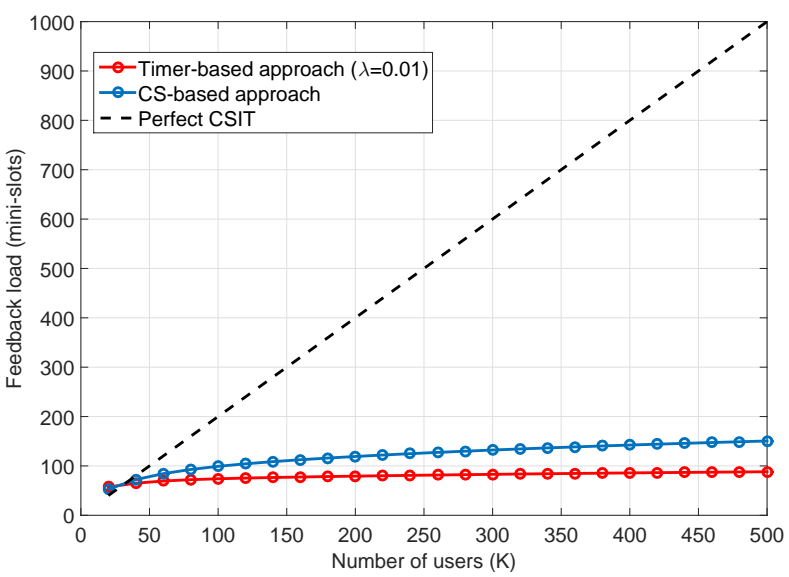

Figure 3. Feedback load versus the number of users $K$.

\section{REFERENCES}

[1] G. Caire, S. A. Ramprashad, H. C. Papadopoulos, C. Pepin, and C.- E. W. Sundberg, "Multiuser MIMO downlink with limited inter-cell cooperation: Approximate Interference Alignment in Time, Frequency and Space," in Proc. 46th Allerton Conf. Commun., Control and Computing,(Monticello, IL), Oct. 2008 .

[2] T. Yoo and A. Goldsmith, "On the optimality of multiantenna broadcast scheduling using zero-forcing beamforming," IEEE Journal on Selected Areas in Communications, vol. 24, pp.528-541, Mar. 2006.

[3] T. Yoo, N. Jindal and A. Goldsmith, "Finite-Rate Feedback MIMO Broadcast Channels with a Large Number of Users," in Proc. IEEE ISIT, July. 2006.

[4] T. Yoo and A. Goldsmith , "Sum-rate optimal multi-antenna downlink beamforming strategy based on clique search," in Proc. IEEE Globecom, Nov. 2005, pp. 1510-1514.

[5] A. Bletsas, A. Khisti, D. Reed and A. Lippman, " A Simple Cooperative Diversity Method Based on Network Path Selection, "IEEE Journal on Selected Areas in Communications, vol. 24, pp. 659-672, March 2006.

[6] K. Elkhalil, M. Eltayeb, H. Shibli, H. R. Bahrami and T. Y. AlNaffouri, "Opportunistic Relay Selection in Multicast Relay Networks using Compressive Sensing," IEEE Globecom Conference, 2014, Dec. 2014, Austin, TX.

[7] M. Eltayeb, T. Al-Naffouri, and H. Bahrami,"Compressive Sensing for Feedback Reduction in MIMO Broadcast Channels," IEEE Transactions on Communications, vol.62, no.9, pp.3209-3222, Sept. 2014.

[8] R. Baraniuk, V. Cevher, M. Duarte and C. Hegde, " Model-Based Compressive Sensing" IEEE Transactions On Information Theory, VOL. 56, NO. 4, April 2010. 